\title{
Returning to orthopaedic business as usual after COVID-19: strategies and options
}

\author{
Francesca de Caro $^{1}$ (1) $\cdot$ Thomas Michael Hirschmann ${ }^{2,3} \cdot$ Peter Verdonk $^{4,5}$
}

Received: 20 April 2020 / Accepted: 22 April 2020 / Published online: 27 April 2020

(c) European Society of Sports Traumatology, Knee Surgery, Arthroscopy (ESSKA) 2020

\begin{abstract}
Purpose The aim of this manuscript is to review the available strategies in the international literature to efficiently and safely return to both normal orthopaedic surgical activities and to normal outpatient clinical activities in the aftermath of a large epidemic or pandemic. This information would be beneficial to adequately reorganize outpatient clinics and hospitals to provide the highest possible level of orthopaedic care to our patients in a safe and efficient manner.

Methods A literature search was performed for relevant research articles. In addition, the World Health Organisation (WHO), the US Centers for Disease Control (CDC), American Association of Orthopaedic Surgeons (AAOS), the EU CDC and other government health agency websites were searched for any relevant information. In particular, interest was paid to strategies and advise on managing the orthopaedic patient flow during outpatient clinics as well as surgical procedures including the necessary safety measures, while still providing a high-quality patient experience. The obtained information is provided as a narrative review.

Results There was not any specific literature concerning the organization of an outpatient clinic and surgical activities and the particular challenges in dealing with a high-volume practice, in the afterwave of a pandemic.

Conclusion As the COVID-19 crisis has abruptly halted most of the orthopaedic activities both in the outpatient clinic and the operating room, a progressive start-up scenario needs to be planned. The exact timing largely depends on factors outside of our control. After restrictions will be lifted, clinical and surgical volume will progressively increase. This paper offers key points and possible strategies to provide the highest level of safety to both the orthopaedic patient and the orthopaedic team including administrative staff and nurses, during the start-up phase.
\end{abstract}

Level of evidence Review, Level V.

Keywords Coronavirus · COVID · Orthopaedic surgery · Outpatient clinic

Francesca de Caro

info@francescadecaro.it

Thomas Michael Hirschmann

Michael.Hirschmann@unibas.ch

Peter Verdonk

pverdonk@yahoo.com

1 Department of Orthopaedic Surgery, Istituto Di Cura Città Di Pavia, Via Parco Vecchio, 27, 27100 Pavia, Italy

2 Department of Orthopaedic Surgery and Traumatology, Kantonsspital Baselland and and University of Basel (BruderholzLiestalLaufen), 4101 Bruderholz, Switzerland

3 University of Basel, 4051 Basel, Switzerland

4 Orthoca, Antwerp, Belgium

5 Antwerp University, Antwerp, Belgium

\section{Introduction}

The current coronavirus pandemic (severe acute respiratory syndrome coronavirus 2-Sars-Cov-2) has impacted all national health care systems within the European community. As a result, many European governments have ordered the shutdown of all elective surgeries and outpatient visits, and a rearrangement of certain orthopaedic departments into COVID-19 reference departments or hospitals to be able to provide both urgent and non-urgent medical treatment to the overwhelming numbers of infected patients [11, 12]. Nevertheless, the infection curve with a steep rise has placed extreme demands on the various health care systems and has led unfortunately to the break down of health care system in certain areas, forcing agonizing choices between live-saving treatment or not for certain patients. 
Over the last few weeks, the strategies to prevent virus spreading have become clear [6]: proper hygiene, quarantine, social distancing, self-isolation and community containment. These main strategies have been able to effectively 'flatten the curve' of COVID-19 infections and hence delay the time of peak infection. However, these strategies do not reduce the absolute number of infections, they merely spread that absolutely number over time and thus provide more valuable time to treat the total number of patients effectively. There is a general consensus that this first wave of COVID19 pandemic, most likely will be followed by a second and/ or third wave of infections in the absence of a vaccine. Thus, on one hand, as the first wave of this pandemic becomes controllable, continued vigilance is imperative and strategies need to be implemented to provide optimal prevention of a resurgence of infection and to react adequately in case of such a resurgence. On the other hand, a progressive return to normal orthopaedic activities is desirable as many patients with semi-urgent or chronic disabilities need help as they have been delayed for a significant period.

As Europe has been spared from similar devastating and disruptive pandemic infections in the last decades, little experience in dealing with these after-effects, and in particular with the return to normal clinical activities, is present within the European health care systems.

The aim of this manuscript is to review the available strategies in the international literature to efficiently and safely return to both normal orthopaedic surgical activities and to normal outpatient clinical activities in the aftermath of a large epidemic or pandemic. This information would be beneficial to adequately reorganize outpatient clinics and hospitals to provide the highest possible level of orthopaedic care to our patients, in a safe and efficient manner.

\section{Methods}

A literature search was performed for relevant research articles. In addition, the World Health Organisation (WHO), the US Centers for Disease Control (CDC), American Association of Orthopaedic Surgeons (AAOS), the EU CDC and other government health agency websites were searched for any relevant information on this topic. In particular, interest was paid to strategies and advise on managing the orthopaedic patient flow during outpatient clinics as well as surgical procedures including the necessary safety measures, while still providing a high-quality patient experience.

As there is an enormous heterogeneity of articles and studies about COVID-19, but only a paucity of articles dealing with the topic of how to return to a normal orthopaedic clinical work, it was recognized that a systematic review is not possible.
Hence, the obtained information is provided as a narrative review stating the current understanding of the virus, the disease, and the counter measures and organizational changes that can be applied in outpatient clinics and orthopaedic surgery in a startup scenario once restrictions are progressively lifted.

\section{Results}

There was not any specific literature concerning the organization of an outpatient clinic and the particular challenges in dealing with a high number of patients in a busy practice, in the afterwave of the COVID-19 pandemic.

Only one article specifically addressing how the novel Coronavirus has affected the orthopaedic surgery during lockdown and how crucial orthopaedic surgeons might be in reining this pandemic, was found [2].

No specific guidelines have yet been developed and shared on the formerly nominated websites and agencies. Considering the lack of direct evidence for this newly identified 2019-nCoV infection, neither systematic review of the literature nor statistics was performed, and the possible strategies and options with this regard are presented. Two specific questions were then addressed:

1. return to orthopaedic business as usual in the outpatient clinic;

2. return to orthopaedic business as usual in the OR.

\section{Discussion}

\section{Return to orthopaedic business as usual in the outpatient clinic}

The basic principle to prevent contamination is to avoid being exposed to the virus. To do so, certain countermeasures can easily be implemented into the daily routine of an outpatient clinic by the orthopaedic surgeon, the administrative and nursery staff. It is of equal importance to create a similarly high level of awareness and responsibility amongst the patient population to adhere to these measures. On the other hand, the orthopaedic team must realise that a significant proportion of the patient population might be reluctant to come to clinics and hospitals for a longer period of time in the aftermath of the pandemic, just out of fear for contamination.

In the absence of community-wide testing, a multi-level gated approach is necessary to ensure the highest level of safety and efficiency possible, for both the patient population and the orthopaedic team (Table 1). 
Table 1 Multilevel approach to clinics

\begin{tabular}{ll}
\hline LEVEL 0 & Screening of symptoms and testing of orthopaedic team (including physicians, nurses, administrative staff) \\
LEVEL 1 & Routine self-questionnaire that routes the patients to the most convenient time slot \\
LEVEL 2 & Temperature controls entering the clinic, symptomatic patients (cough, dyspnea) to be sent to emergency; \\
& surgical masks provided to each patient \\
LEVEL 3 & Maintain social distancing, no crowded waiting rooms, patients waiting in the car until they are called in \\
LEVEL 4 & Clinical visit made efficiently and swiftly, subjective history digitally recorded before entering room \\
LEVEL 5 & Digital scheduling of follow-up visits and payment avoiding exchange of documents \\
\hline
\end{tabular}

LEVEL 0. Reopening of the orthopaedic outpatient clinic should ideally be associated with a screening for symptoms and testing of the entire orthopaedic team including physicians, nurses and administrative staff 1 day ahead of the opening. If tested negative, repeat testing at specific intervals might be advisable for screening and documentation purposes. If tested positive, the person should be quarantined and retested 14 days later. As reinfection and reactivation rates of the virus are still unclear, continued vigilance and repeat testing are still warranted.

LEVEL 1. As a significant number of patients might be asymptomatic and infectious [4], a routine questionnaire identifying potential infection or high risk for infection is currently being developed in collaboration with the CDC, Apple and Google. These self-questionnaires can be found at https://www.apple.com/covid19/ and include questions on the presence of emergency symptoms (severe, constant chest pain or pressure; extreme difficulty breathing; severe, constant lightheadedness; serious disorientation or unresponsiveness), age group (below 18, 18-64, above 65), recent symptoms (fever, chills or sweating; difficulty breathing; new or worsening cough; sore throat; aching throughout the body; vomiting or diarrhea), underlying disease or risk factors, international travel in the last 14 days, COVID-19 incidence in your area, direct exposure to others with COVID in the last 14 days, living in a long-term care facility, working in a medical facility in the last 14 days. This questionnaire is definitely of interest once the current restrictions are progressively lifted and could provide the orthopaedic team some guidance in getting specific categories of patients back into the office.

As the elderly and patients with co-morbidities are at a higher risk for nosocomial transmission [3], it could be of interest to start up your clinic with the younger asymptomatic patients without documented risk factors. Ideally, the questionnaire section relating to specific COVID symptoms is performed digitally, 1 day ahead of the outpatient visit. Age, comorbidities and risk factors could be collected at the moment the patient is making the appointment. This information could route the patient to the most convenient time slot taking into account that older patients or patients with comorbidities are advised to wait until a later stage after restart.
LEVEL 2. Upon entering the clinic, temperature controls using individual temperature checks by a certified nurse or by infrared cameras might further allow identification of patients with fever. Obvious observations like sweating, dyspnea or coughing need to draw the attention and alarm the staff for a potential infection. Patients need to be provided with a single-use face mask or surgical mask to reduce the risk of spreading virus particles. Usually no aerosol-producing procedures are performed during outpatient clinic, so the need of more advanced protective masks, such as FFP2 (N95) masks, should be not recommended for the medical doctors and sanitary personnel [5]. The reception staff needs to be protected adequately by avoiding unnecessary direct and indirect contact with patients. Ideally, exchanging any type of physical document or paperwork (ID card, written questionnaires) should be avoided. If impossible to avoid, adequate decontamination is necessary.

LEVEL 3. Waiting areas will need specific attention including social distancing and hygiene. These countermeasures will significantly reduce the capacity of the waiting area and will profoundly affect the patient flow. The corona crisis will force the orthopedic team to redesign and rethink waiting areas. Fully packed waiting areas will no longer be the ideal and recommended scenario. Patients ideally wait outside, in the car, in the parking lot, in the cafeteria and are called in just in time. In between patients, the seats and other furniture need to be disinfected routinely.

LEVEL 4. Once entering the examination room or doctor's office, access to and from the examination office space might be facilitated; conversion of a hand knob to an elbow knob might mitigate the contamination risk. The time spent in the room could be strictly minimalized in time and human resources. Therefore, certain aspects of the clinical visit like the subjective history taking might be done prior to the visit using a digital interface. The clinical orthopaedic exam should be done swiftly and efficiently. Orthopaedic residents and fellows are allowed to participate in the process.

Often, prior to the corona era, surgery booking was performed in a different room. To reduce cross contamination between patients, it is advised to have the patient remaining in the same office and have the person responsible for the surgery book come to that same room. 
Once the clinical visit has ended, the patient leaves the office. The office and all surfaces and objects should be decontaminated. This includes tables, doorknobs, light switches, countertops, handles, desks, phones, keyboards.

LEVEL 5. Post-visit scheduling of follow-up appointment and payments often requires an additional visit to the registration desk. The interaction between the administrative personal and the patient needs to be minimized avoiding exchange of documents, cash money, and physical interaction with hospital devices such as the keyboard of the ATM machine.

It now has become clear that outpatient visits will be significantly affected both in capacity and safety measures. Patient waiting areas will be limited and necessitate a new organizational model similar to an industrial just-in-time delivery approach. As restrictions are progressively lifted, older patients and patients with co-morbidities should be kept away initially. Adequately filtering and routing these patients is another point of attention and should be done pre-emptively.

\section{Return to orthopaedic business as usual in the OR}

Orthopaedic surgery (excluding traumatology) in the early days after the lockdown should still be applied judiciously. The decisions on the pathway to business as usual for orthopaedic surgery should be made by a panel including the head of the hospital, the chief of the ICU, chief of anesthesia and chief of orthopaedic surgery.

Upon condition that all resources (personal protective equipment (PPE), intensive care unit (ICU) beds, medication, respirators, personnel, OR capacity, anticipated length of stay) are available, a progressive surgical start-up can be anticipated (Table 2).

Providing adequate information to patients with regards to specific hospital organization for infection prevention, is an important step in going back to surgery. It should be anticipated that a significant part of the patient population will consider the hospital as a high-risk area for infection. Therefore, this information is crucial to re-establish a stable patient-hospital relation based on trust and confidence.

There is a general consensus that all patients should be screened for symptoms and tested 1 day prior to surgery. It is, however, also very important to understand that all available tests have a significant false-negative rate, which could result in a false-safety feeling!

At the moment, the RT PCR swab testing is $100 \%$ specific, but is limited in terms of sensitivity. The naso-pharingeal (NP) swab test seems to have a better accuracy than the oro-pharingeal (OP) test, with the SARS-CoV-2 RNA detected only in $32 \%$ of OP swabs, which was significantly lower than that in NP swabs (63\%). The technique is easy, well accepted from the patient, with low risk of infection of the sanitary personnel actually doing the testing [9]. The supine position during the testing is recommended but not mandatory. However, universal availability is still limited in certain areas and the turnaround times can be lengthy.

Rapid diagnostic tests (RDTs) immunoassays and serology procedures, are small stand-alone tests, simple to use by minimally trained staff at organized points of care (the site of triage or outside the hospital). They provide test results within $15 \mathrm{~min}$. They can be antigen or antibodies detecting tests, for the antibodies it must be kept in mind that it takes a few and up to 10 days before the concentration of antibodies in the blood is high enough to be captured by the RDT [9, $10,13]$. These tests are not validated at the moment, they do have CE marking, they are not already recommended for case detection, playing a role in research and surveillance at the moment but with a growing interest from scientists and surgeons as they are attractive for decentralized testing particularly in low resource settings.

Moreover, patients undergoing surgery could be tested by CT scan. Some radiology literature suggests a pivotal role for CT as a speedy and readily available option, especially with shortages of the PCT test, which is more time consuming. The overall specificity and sensitivity are largely depending on the incidence of disease and ranges between 61 and $97 \%$ with a reported false-negative rate up to $20 \%$ in symptomatic patients [1,7].

For every negatively tested patient, it is advised to convert general anesthesia, which is an aerosol-generating procedure, to local or locoregional anesthesia as much as possible, to avoid cross contamination of the anesthesia team and to reduce overuse of the available resources. Outpatient or short-stay surgery should be promoted to reduce the risk of nosocomial infection.

Positively tested patients should not be operated but should be delayed for a minimal period of 3 weeks and need
Table 2 Key points for approaching surgeries

\author{
Screening of patients day before of surgery (NP Swab, Serological Test, CT Scan) \\ Definition of "Urgent", and "Urgent, Somewhat Elective Surgery" \\ Favour day surgical cases (requiring $\leq 24 \mathrm{~h}$ of hospital stay), younger patients or ASA $1-2$ older patients \\ If major surgery (I.E total joint replacement) is needed, strictly apply fast-track protocols \\ Avoid general anesthesia and all aerosol-generating procedures \\ Negative pressure rooms and strict sanification after each patient to be required
}


two confirmed negative PCR swab test with a $24 \mathrm{~h}$ interval (personal communication with Bergamo Italy COVID team).

General definitions of what constitutes the necessity for surgery (elective; urgent, somewhat elective; urgent) are offered from a variety of sources and can serve as an additional guide to schedule these delayed cases. In the progressive start-up phase, 'urgent' and 'urgent, somewhat elective' surgery might be considered. Elective surgery should be further delayed until the highest level of safety can be guaranteed. According to AAOS guidelines, patients for whom surgery is deemed "elective" are those with chronic problems whose surgery can certainly be delayed without significant harm to the patient or eventual outcome. Although an argument can be made for the need for surgery in some individuals due to pain or functional impairment, the determining principle is that delaying treatment will not significantly alter the eventual outcome. Such surgeries include elective total joint replacements for osteoarthritis, spinal fusion, chronic joint conditions (e.g., atraumatic, chronic rotator cuff tears; posterior cruciate ligament injuries; and degenerative meniscal tears) and other conditions that although painful will not be altered by delay in treatment (e.g., elbow tendinitis and carpal tunnel surgery). Whereas the pathology may cause significant functional impairment to the patient, severely reducing his quality of life, major surgeries such as total joint replacement should still be performed. Patients undergoing total joint replacement will need to be able to be discharged from the hospital in the shortest delay possible (generally less than $72 \mathrm{~h}$ ), thus to reduce the risk of nosocomial infections $[2,8]$. Some outpatient surgery can be considered in early start-up phase, as the pathology can become more complex if left untreated or associated with a major functional deficit (defined as urgent, somewhat elective). Surgery for injuries such as anterior cruciate ligament tears, locked or bucket handle meniscus, acute traumatic rotator cuff tears, biceps injuries, should still be performed. If possible, these procedures should be done in the outpatient setting to minimize utilization of resources.

Standard PPE (surgical mask) must be provided to every single patient entering the OR. During orthopedic surgeries, frequent surgical aerosol procedures are performed such as the use of high-speed power tools, electro-cautering and pulsed irrigation (even if not recommended). Thus, the use of correct PPE, including surgical gloves, a water-resistant surgical gown with long sleeves, a surgical mask, and fullface protection with a face-shield and FPP2 masks is recommended [5].

Basically, it would be reasonable to restart surgery (urgent and urgent, somewhat elective) activities with day surgical cases (requiring $\leq 24 \mathrm{~h}$ of hospital stay) using preferably local or loco-regional anesthesia. This includes mostly arthroscopic surgery (shoulders, knees and ankles) and simple day procedures. Reducing length of stay means reducing their risk for nosocomial COVID-19 infections. Moreover, given their relatively short hospital stays, this kind of surgeries do not entail a major drain on health-care resources. Finally, hospital beds can still be freed up quickly, if required, for any emergency admissions due to a new infection outbreak [2].

Older patients affected by more than one comorbidity, or overweight patients, that are the ideal patients needing a total joint replacement, are commonly known to be at higher risk of developing severe acute respiratory syndrome coronavirus 2-Sars-Cov-2 [3]. Thus, it is reasonable, in a first moment, to postpone this kind of surgery, trying to create an ad hoc outpatient path for higher risk patients and giving them all ortho-biologic treatments that knowingly can delay the implantation of a joint replacement.

As we have previously highlighted, general anesthesia should be avoided, thus reducing the risk of infection of the sanitary personnel and limiting the risk of transmission to the patients. Operating rooms should be equipped with negative pressure and no recycle used. All aerosol-producing equipment should be banned, the use for example of a suction electrocautery should be provided in every operating room.

Once the patient has departed from the OR, the room should be left empty for a specific period and all high-touch surfaces including the anesthesia machine and the anesthesia work area should be cleaned and disinfected with an Environmental Protection Agency (EPA)-approved hospital disinfectant. The length of time in between patients depends on the number of air exchanges per hour in the specific room or space. More detailed guidance is available from the Centers for Disease Control and Prevention (CDC).

\section{Conclusion}

As the corona crisis has abruptly halted most of the orthopaedic activities both in the outpatient clinic and the operating room, a progressive start-up scenario needs to be planned. The exact timing largely depends on factors outside of our control. After restrictions will be lifted, clinical and surgical volume will progressively increase. This paper offers some rules of thumb and possible strategies to provide the highest level of safety to both the orthopaedic patient and the orthopaedic team including administrative staff and nurses, during the start-up phase.

\section{Compliance with ethical standards}

Conflict of interest Authors declare no conflict of interest.

Funding No funding is provided in this study. 
Ethical approval This article does not contain any studies with human or animals performed by any of the authors.

Informed Consent For this type of study, formal consent is not required.

\section{References}

1. Ai T, Yang Z, Hou H, Zhan C, Chen C, Lv W et al (2020) Correlation of chest CT and RT-PCR testing in coronavirus disease 2019 (COVID-19) in China: a report of 1014 cases. Radiology. https:// doi.org/10.1148/radiol.2020200642

2. Chang LZ, Wang W, Murphy D, Po Hui JH (2020) Novel coronavirus and orthopaedic surgery: early experiences from Singapore. J Bone Jt Surg Am. https://doi.org/10.2106/JBJS.20.00236

3. Chen T, Wu D, Chen H, Yan W, Yang D, Chen G (2019) Clinical characteristics of 113 deceased patients with coronavirus disease 2019: retrospective study. BMJ 368:m1091. https://doi. org/10.1136/bmj.m1091

4. Day M (2020) Covid-19: four fifths of cases are asymptomatic, China figures indicate. BMJ 369:m1375. https://doi.org/10.1136/ bmj.m1375

5. Hirschmann MT, Hart A, Henkel J, Sadoghi P, Seil R, Mouton C (2020) COVID-19 coronavirus: recommended personal protective equipment for the orthopaedic and trauma surgeon. Knee Surg Sports Traumatol Arthrosc. https://doi.org/10.1007/s00167-02006022-4 (Article in press)

6. Hou C, Chen J, Zhou HL, Yuan J, He S et al (2020) The effectiveness of the quarantine of Wuhan city against the corona virus disease 2019 (COVID-19): well-mixed SEIR model analysis. J Med Virol. https://doi.org/10.1002/jmv.25827
7. Inui S, Jujikawa A, Jitsu M, Kunishima M, Watanabe S, Suzuki $\mathrm{Y}$ et al (2020) Chest CT findings in cases from the cruise ship "Diamond Princess" with coronavirus disease 2019 (COVID-19). Radiol Cardiothorac Imaging. https://doi.org/10.1148/ryct202020 0110

8. Jansen JA, Kruidenier J, Be SpeK, Snoeker BAM (2020) A costeffectiveness analysis after implementation of a fast-track protocol for total knee arthroplasty. Knee. https://doi.org/10.1016/j. knee.2019.09.014

9. Loeffelholz MJ, Tang YW (2020) Laboratory diagnosis of emerging human coronavirus infections-the state of the art. Emerg Microbes Infect 9(1):747-756. https://doi.org/10.1080/22221 751.2020.1745095

10. Pang J, Wang MX, Ang IYH, Tan SHX, Lewis RF, Chen JI et al (2020) Potential rapid diagnostics, vaccine and therapeutics for 2019 novel coronavirus (2019-nCoV): a systematic review. J Clin Med 9(3):623. https://doi.org/10.3390/jcm9030623

11. Rajan N, Joshi GP (2020) The COVID-19: role of ambulatory surgery facilities in this global pandemic. Anesth Analg. https:// doi.org/10.1213/ANE.0000000000004847

12. Spina S, Marrazzo F, Migliari M, Stucchi R, Sforza A, Fumagalli R (2020) The response of Milan's Emergency Medical System to the COVID-19 outbreak in Italy. Lancet 395(10227):e49-e50. https://doi.org/10.1016/S0140-6736(20)30493-1

13. Zhao J, Yuan Q, Wang H, Liu W, Liao X, Su Y et al (2019) Antibody responses to SARS-CoV-2 in patients of novel coronavirus disease. Clin Infect Dis. https://doi.org/10.1093/cid/ciaa344

Publisher's Note Springer Nature remains neutral with regard to jurisdictional claims in published maps and institutional affiliations. 